\title{
A Broad-Band LTCC Integrated Transition of Laminated Waveguide to Air-Filled Waveguide for Millimeter-Wave Applications
}

\author{
Yong Huang and Ke-Li Wu, Senior Member, IEEE
}

\begin{abstract}
In this paper, a compact and broad-band integrated transition between laminated waveguide in a multilayer low-temperature co-fired ceramic (LTCC) substrate and standard air-filled rectangular waveguide is presented. A parallel inter-coupled two-pole filter equivalent circuit is employed to interpret the working mechanism of the transition and to predict the performance. A $K a$-band prototype of the proposed broad-band transition is designed and fabricated in an LTCC substrate. The simulated and measured results of the prototyped transition show good agreement. It has been demonstrated, through the experimental results of the $K a$-band prototype that the proposed transition configuration gives an effective bandwidth of over $8 \%$ with $-15-\mathrm{dB}$ return loss and average $-0.4-\mathrm{dB}$ insertion loss over the bandwidth at the $K a$ frequency band.
\end{abstract}

Index Terms-Laminated waveguide, low-temperature co-fired ceramic (LTCC), millimeter wave, rectangular waveguide (RWG), waveguide transition.

\section{INTRODUCTION}

$\mathbf{L}$ OW-TEMPERATURE co-fired ceramic (LTCC) technology offers many advantages to microwave and millimeter-wave applications, such as low losses, high-density integration, and low cost for high-volume production. In addition to a wide range of applications of the technology in RF modules, the development of the laminated waveguide concept [1] by making use of LTCC in millimeter-wave frequency ranges has called tremendous attentions to the engineers in the field. A recent notable work is the grid-like reinforced conducting sidewall structure of the LTCC laminated waveguide using multilayer conducting strips [2] and transitions from the waveguide to various integrated transmission lines [3].

An effective three-dimensional (3-D) laminated waveguide is constructed by depositing metal planes on the top and bottom surfaces of a multilayered substrate and using grid-like conductive walls as sidewalls. The grid-like reinforced conductive sidewalls in the laminated waveguide comprise a plurality of filled through via-holes disposed at predetermined intervals and a plurality of sub-conductor layers deposited between layers of the dielectric substrate so as to electrically connect the filled viaholes together inside the dielectric substrate. Due to its negligible radiation and low transmission losses, the laminated waveguide is considered as a high-performance transmission line for

Manuscript received October 15, 2002; revised December 23, 2002. This work was supported by the Research Grants Council of the Hong Kong Special Administrative Region under Grant 2150279.

The authors are with the Department of Electronics Engineering, The Chinese University of Hong Kong, Shatin, Hong Kong (e-mail: klwu@ee.cuhk.edu.hk).

Digital Object Identifier 10.1109/TMTT.2003.810146 integrated millimeter-wave applications such as integrated antenna array for collision-avoidance radar [4], [5]. A laminated waveguide can also be used to design some integrated passive components such as a miniaturized ridge waveguide filter [6], [7]. Nevertheless, in many practical applications, an integrated laminated waveguide module is only a part of a system; it needs to be interfaced with other types of transmission lines. Among them, a conventional air-filled waveguide would likely be a popular one. When a laminated waveguide module is to be connected to an air-filled waveguide in a system, an effective transition between the two different types of transmission line is required.

Air-filled rectangular waveguides (RWGs), due to their excellent electric performance, are still essential components in many practical millimeter-wave systems such as the local multipoint distributed system (LMDS). An effective transition that connects a laminated waveguide module with an RWG system would be a compulsory and critical part for overall system design. Since the cross section of a laminated waveguide is much smaller than that of a RWG, due to the high dielectric constant (approximately 7-10) of the filled substrate and the thickness limitation of the laminated waveguide, there is a large dimension mismatch between the two types of waveguides. The mismatch causes tremendous difficulties in impedance matching and energy losses. Therefore, designing a broad-band and compact transition between the two types of waveguides is a fundamental and challenging problem.

Although transition configurations of laminated waveguides to various practical transmission lines, including microstrips/strip lines [3], [8] and coplanar waveguides [3], [9], have been recently reported, to the best knowledge of the authors, no configurations have ever been proposed until now for a laminated waveguide to air-filled waveguide transition.

In this paper, a broad-band integrated laminated waveguide to air-filled waveguide transition is proposed. A prototype of an integrated $K a$-band transition between an LTCC laminated waveguide (using Dupont 943 Green Tape) and WR28 waveguide was designed and fabricated for proving the concept. A 2.5-GHz bandwidth defined at $-15-\mathrm{dB}$ return loss is obtained for the transition at a center frequency of $29 \mathrm{GHz}$. The experimental result correlates well with the electromagnetic (EM) designed performance. An average insertion loss of $-0.4 \mathrm{~dB}$ is observed from the experimental results for the prototyped transition, demonstrating its promising low-loss and broad-band features. Such a transition provides a broad-band, compact, and low-cost interface for LTCC integrated laminated waveguide modules. 


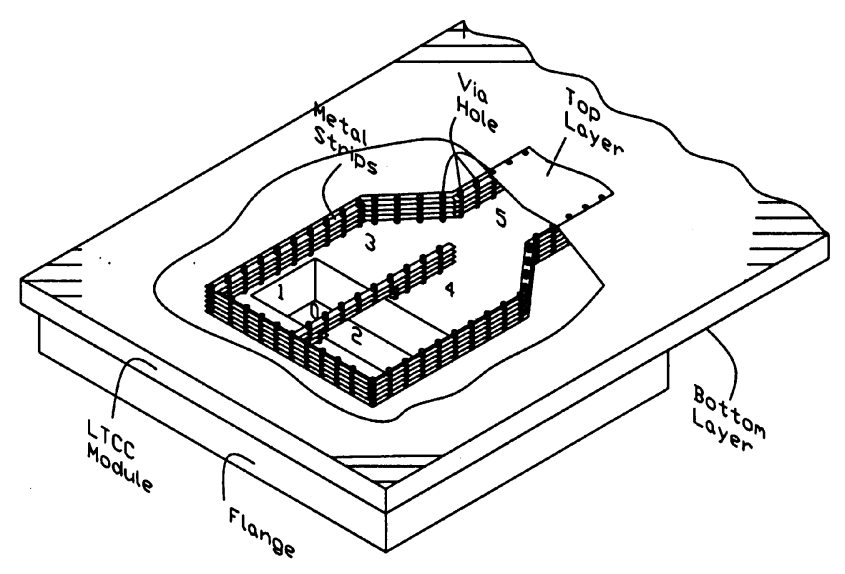

Fig. 1. Structure of the laminated waveguide to air-filled waveguide transition.

\section{Configuration of the Transition AND Its WORKING MECHANISM}

Fig. 1 shows the 3-D structure of the proposed multilayered transition. The top and bottom layers of the LTCC module are fully covered by metal, except a rectangular aperture on the bottom layer. The aperture that is called the input coupling aperture thereafter has the same size as the inner cross section of the air-filled waveguide connected to the LTCC laminated waveguide module. The microwave energy is transferred between an air-filled waveguide and laminated waveguide regions through the input-coupling aperture. As shown in Fig. 1, inside the LTCC module, separated by a partition conductive wall, two parallel laminated waveguides are formed and excited by the input-coupling aperture. The two laminated waveguides are shorted at one end and connected to a single laminated waveguide port on the other end through a $Y$ branch structure. In Fig. 1, 0 and 5 denote the air-filled waveguide and the single laminated waveguide, respectively, and 1-4 denote four equivalent resonators, which will be explained below in detail, inside the shielded transition region.

Being filled by a high-permittivity LTCC substrate, and being shielded by conductive top and bottom layers, as well as conductive via-hole walls, two parallel resonator chains, which are separated by the partition wall, are effectively constructed inside the transition. As shown in Fig. 1, each resonator chain effectively comprises two series connected quasi-half-wavelength resonators. The definition of each of equivalent resonators and the associated variables of the transition are shown in Fig. 2 for convenience of the discussion.

In Fig. 2, the rectangle frame with length $a$ and width $b$ denotes the input-coupling aperture. From the shorting wall $\mathrm{C}-\mathrm{C}^{\prime}$ to the reference line $\mathrm{B}-\mathrm{B}^{\prime}$, a pair of quasi-half-wavelength waveguide resonators denoted as 1 and 2 are formed. These two resonators are excited in phase by the input-coupling aperture and are inter-coupled through a mutual coupling aperture located on the partition wall. By removing part of metal strips on some sub-conductor layers and correlative filled via-holes, the mutual coupling aperture is created on the partition wall. The mutual coupling aperture with height $h$ and length $b$ is more apparently shown in Fig. 3, which illustrates the side view of the transition along bisection line $\mathrm{D}-\mathrm{D}^{\prime}$. The thickness of the partition wall is denoted as $w$, as shown in Fig. 2. Obviously, $h$ and $w$

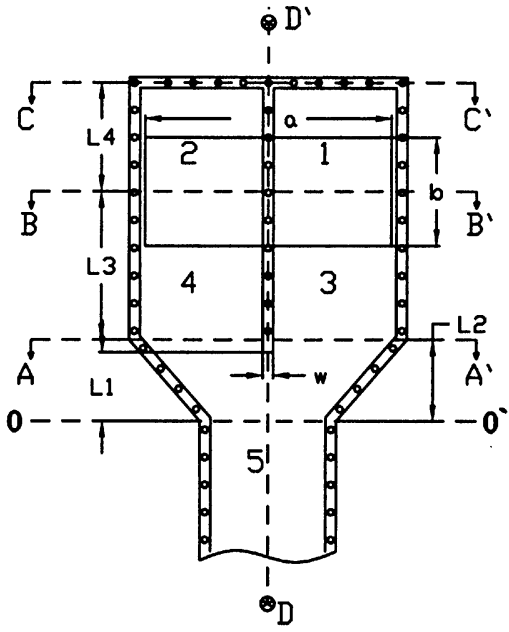

Fig. 2. Top view of the transition and the associate variables.

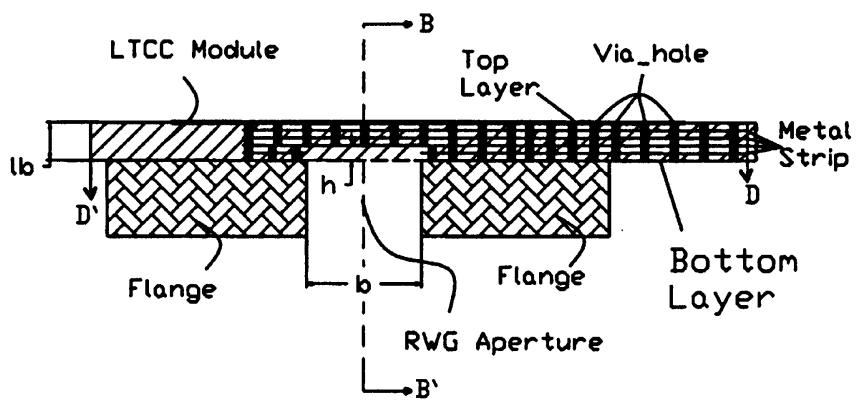

Fig. 3. Side view of the transition along bisection line D-D'.

affect the coupling between the air-filled waveguide and laminate waveguide and also affect the mutual coupling between the resonators inside the LTCC substrate.

Another pair of laminated waveguide resonators denoted as 3 and 4 are formed between reference line $\mathrm{B}^{-\mathrm{B}^{\prime}}$ and the $Y$ branch junction, which also provides a coupling between the two resonators. Again, this pair of resonators are excited by the input-coupling aperture and coupled with each other via the mutual coupling aperture. Note that the couplings between the input coupling aperture and resonators 3 and 4 are negative with respect to those between the input coupling aperture and resonators 1 and 2 because of the nature of the $E$-plan $T$-junction.

According to the coupling relations discussed above, the equivalent-circuit model shown in Fig. 4 can be employed to predict the performance and explain the working mechanism of the transition. In the equivalent circuit, each $L C$ loop represents the corresponding resonator in the transition structure. The coupling coefficients $M_{01}, M_{02}, M_{03}$, and $M_{04}$ denote the couplings between air-filled waveguide and resonators $1-4$, respectively, and the coupling coefficients $M_{35}$ and $M_{45}$ denote the couplings between the laminated waveguide port and resonators 3 and 4, respectively. The coupling coefficients $M_{13}, M_{12}, M_{24}$, and $M_{34}$ signify the mutual coupling between the four resonators. As a matter of fact, due to the symmetry of the transition, a magnetic wall is present in the plane D- $\mathrm{D}^{\prime}$ (Fig. 2). Therefore, no power crosses this plane. Here, the coupling coefficients $M_{12}$ and $M_{34}$ are only used to present the loading effect of the mutual coupling aperture. 


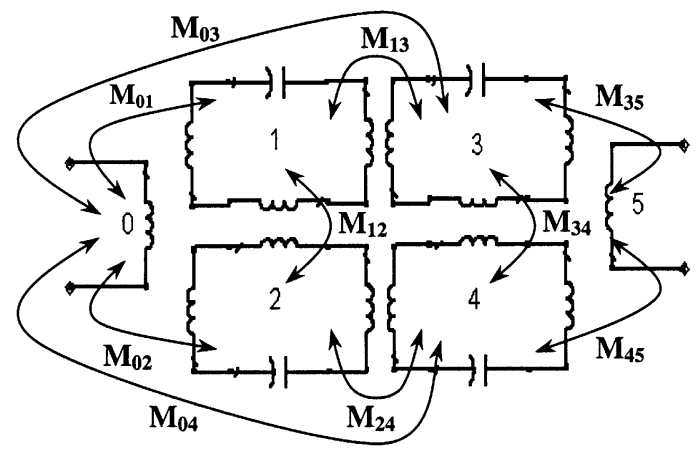

Fig. 4. Equivalent-circuit model of the transition.

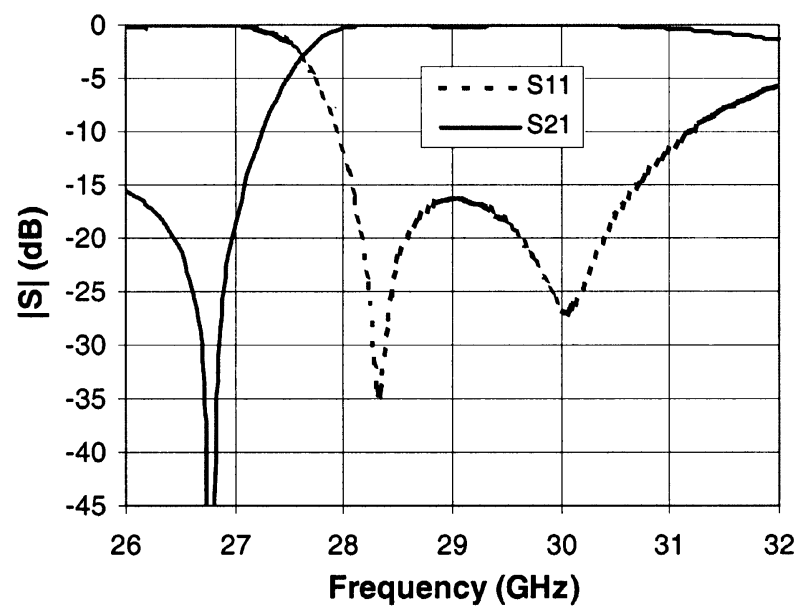

Fig. 5. Simulated performance of the equivalent-circuit model.

As shown in Fig. 4, resonators 1 and 3 and 2 and 4 form a pair of two-pole filters, respectively. According to the circuit model, there will be two reflection zeros within the passband and a transmission zero in the finite frequency region. The finite transmission zero is caused by the cross-couplings of $M_{03}$ and $M_{04}$. Due to the symmetry of the structure along D- $\mathrm{D}^{\prime}$, it is obvious that $M_{13}=M_{24}, M_{01}=M_{02}, M_{03}=M_{04}$, and $M_{35}=M_{45}$. By designing the coupling matrix of the equivalent circuit appropriately, a desired passband performance can be obtained. A simulated transition response is shown in Fig. 5 with a specific coupling matrix, in which $M_{01}=M_{02}=0.898$, $M_{03}=M_{04}=-0.714, M_{12}=3.269, M_{34}=0.30, M_{13}=$ $M_{24}=1.797$, and $M_{35}=M_{45}=1.076$. The center frequency and bandwidth used to simulate the response are 29 and $1.6 \mathrm{GHz}$, respectively.

\section{EM Simulation AND DESIGN OF THE TRANSITION}

By comparing the equivalent-circuit model with the physical structure of the transition, one can observe that: 1) changing $h$ can control coupling coefficients $M_{01} \sim M_{04}$, as well as $M_{12}$ and $M_{34}$;2) $L 4$ determines the resonant frequency of resonators 1 and 2; altering $L 4$ can also control the coupling coefficients $M_{13}$ and $M_{24}$, as well as $M_{01}$ and $M_{02}$; and 3) $L 3$ affects the resonant performance of the resonators 3 and 4; tuning $L 3$ can also control the coupling coefficient $M_{03}, M_{04}, M_{13}$, and $M_{24}$. It is obvious that $L 1$ and $L 2$ determine the performance of the $Y$ branch so these two values can be simply designed for an

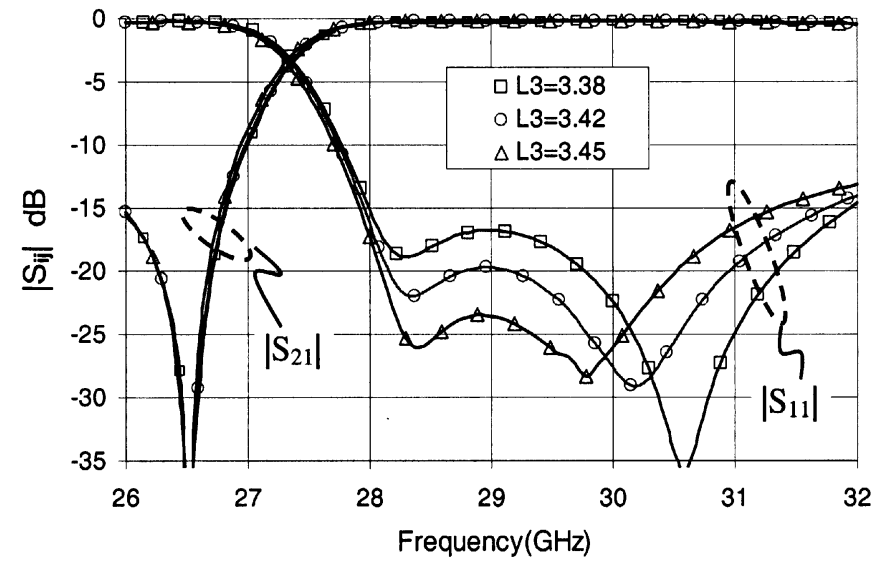

Fig. 6. Effect of $L 3$ (unit: millimeters, other dimensions: $L 1=1.91, L 2=$ $2.03, L 4=2.44, h=0.45$, and $w=0.13)$.

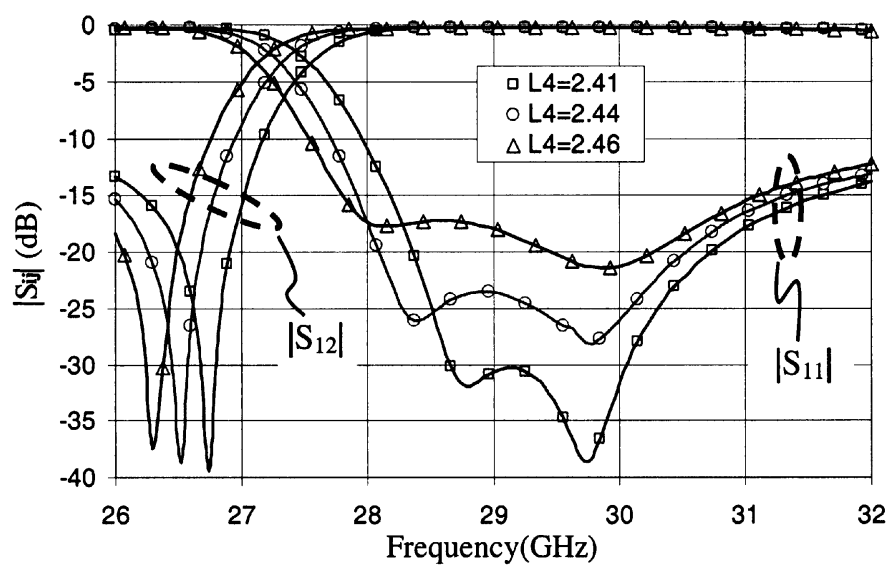

Fig. 7. Effect of $L 4$ (unit: millimeter, other dimensions: $L 1=1.91, L 2=$ $2.03, L 3=3.45, h=0.45$, and $w=0.13$ ).

optimized $Y$ branch. Therefore, design of a transition becomes a matter of designing $L 3, L 4$, and $h$.

In order to determine the dependence of the transition performance on the resonator length $L 3, L 4$ and the height $h$ of the mutual coupling aperture, a numerical parametric study is performed with Ansoft HFSS, a full-wave finite-element method 3-D EM simulator. A WR28 waveguide is used in the study as an air-filled waveguide whose cross-sectional dimension is $7.11 \times 3.56 \mathrm{~mm}^{2}$. The laminated waveguide with a cross-sectional dimension of $3.56 \times 0.89 \mathrm{~mm}^{2}$ is chosen by using eight layers of Dupont 943 Green Tape with $\varepsilon_{r}=7.5$ and $\tan \delta=$ 0.002. A piece of a 3-mm-long air-filled waveguide and a piece of a 5-mm-long laminated waveguide are incorporated in the transition model of the EM simulation. A full conductor wall transition modal is used in the parametric study.

Figs. 6 and 7 show the simulated results of the magnitude of $S$-parameters of the transition by varying $L 3$ and $L 4$, respectively. As can be observed, the two reflection zeros can be separately controlled by $L 3$ and $L 4$. This is because, as the resonator length increase, the corresponding resonating frequency decreases, and its corresponding reflection zero shifts down. This feature can be used to control the bandwidth of the transition.

As interpreted above, adjusting the length of the two pairs of resonators also affects the coupling coefficient $M_{01}-M_{04}$, 


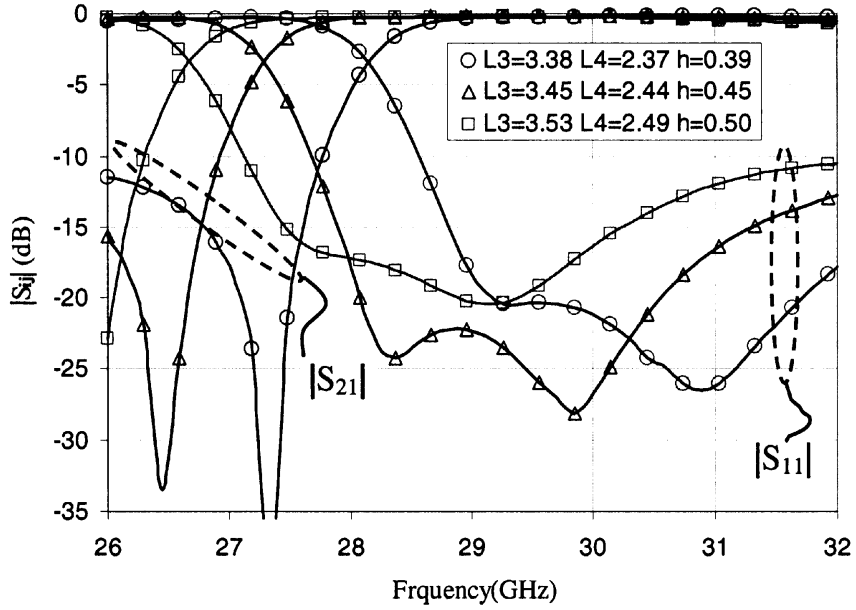

Fig. 8. Design of transition working at different center frequencies. ( $L 1=$ $1.91, L 2=2.03$, and $w=0.13$, unit: millimeters).

which causes a change of the matching at the same time. To obtain a desired passband performance at a predestined center frequency, the height $h$ should be adjusted to cooperate with $L 3$ and $L 4$ to achieve the broadest bandwidth. Fig. 8 shows three passband responses with three groups of $L 3, L 4$, and $h$ values. The center frequency is shifted from 30.5 to $28.5 \mathrm{GHz}$, while all three length increase.

It can be concluded, from the above discussion, that by adjusting $L 3, L 4$, and $h$, one can design a transition with a broad bandwidth at the desired center frequency. The simulated results also reveal that over $11 \%$ bandwidth with a return loss of better than $-15 \mathrm{~dB}$ and an insertion loss of better than $-0.3 \mathrm{~dB}$ can be obtained by the proposed transition structure.

\section{EXPERIMENTAL RESULTS}

A prototype of the proposed transition working in the LMDS frequency band was designed at a center frequency of $29 \mathrm{GHz}$. In order to facilitate the measurement, two testing modules were fabricated and measured to verify the overall performance of the transition. One module is a back-to-back configuration with two laminated waveguide ports of two identical transitions connected with a piece of 14.22-mm-long laminated waveguide in between. Another module is a single transition with an absorbing terminal at the laminated waveguide port. All transition ports of the two modules are interfaced with WR28 waveguides.

The transition modules were built on an eight-layer LTCC tile. Metal strips with a width of $0.14 \mathrm{~mm}$ are employed to connect the via-holes of $0.09 \mathrm{~mm}$ in diameter to form effective conducting waveguide sidewalls. The center-to-center via pitch of the waveguide walls is $0.38 \mathrm{~mm}$. The other dimensions are (unit: mils) $h=0.34, L 4=2.43, L 1=1.77, L 2=1.91$, and $L 3=3.35$. Fig. 9 shows a photograph of the back-to-back transition module and the single transition module. WR28 waveguide flanges have been mounted on the panel to interface the modules.

The $S$-parameters of the transition modules are directly measured using an HP8510C vector network analyzer. A careful thru-reflect line (TRL) calibration was used to move the reference planes to the interface surface of the waveguide flanges. The measured reflection coefficient of the single transition

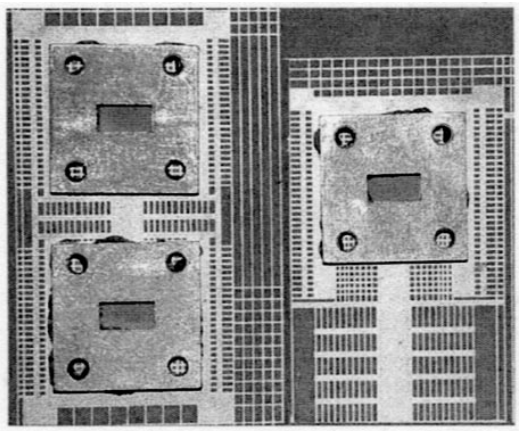

Fig. 9. Photograph of the back-to-back configuration module (left-hand side) and the single transition module (right-hand side) in an LTCC tile (35.2 mil in thickness).

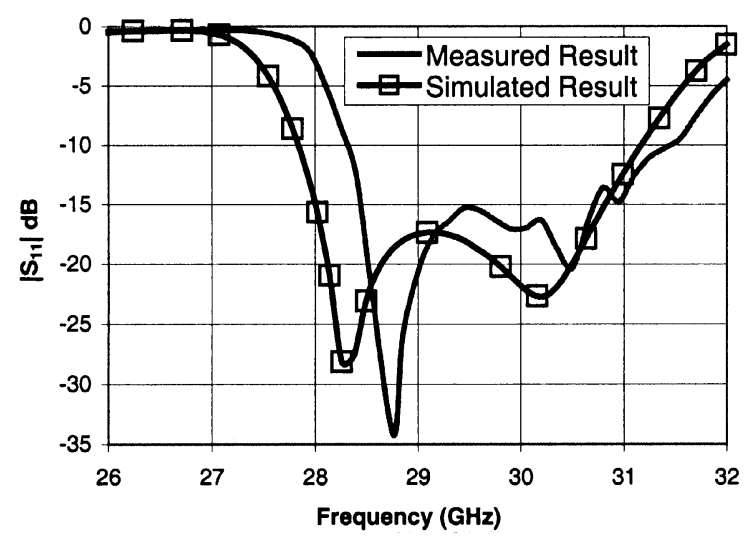

Fig. 10. Measured and EM simulated $|S 11|$ of a single transition.

module is superposed with that of the EM designed structure, in which the actual grid-like conducting sidewalls were used, as shown in Fig. 10. Good correlation between the two results can be observed. The measured bandwidth is approximately $2.5 \mathrm{GHz}$ with a return loss below $-15 \mathrm{~dB}$. A slight frequency shift and a bandwidth reduction might be caused by a slight offset of dimension $L 4$ in the prototype hardware.

Fig. 11(a) shows the comparison of the simulated and measured return losses of the back-to-back module. Good correlation between the measured and EM simulation results are obtained.

The simulated and measured insertion losses of the back-to-back module are shown in Fig. 11(b), which also shows good correlation. The good correlation confirms the validation of the loss model used in the EM simulation and indicates that EM simulation can provide a fair estimation of insertion loss for the transition. As a matter of fact, because of unequal waveguide interfaces of the transition, it is difficult to experimentally characterize the insertion loss of a single transition. Therefore, EM simulated insertion loss, which has been validated indirectly by the back-to-back module, is used to characterize the transition, which is also superposed in Fig. 11(b). It can be observed that the insertion loss of a single transition can be as low as $-0.3 \mathrm{~dB}$ from 28.2 to $31.0 \mathrm{GHz}$. Accounting for a slight underestimation of insertion loss learned from the difference between the simulated and measured results of the back-to-back module, it can be concluded that an average insertion loss of a single transition should be better than $-0.4 \mathrm{~dB}$ in the whole passband. It should be mentioned 


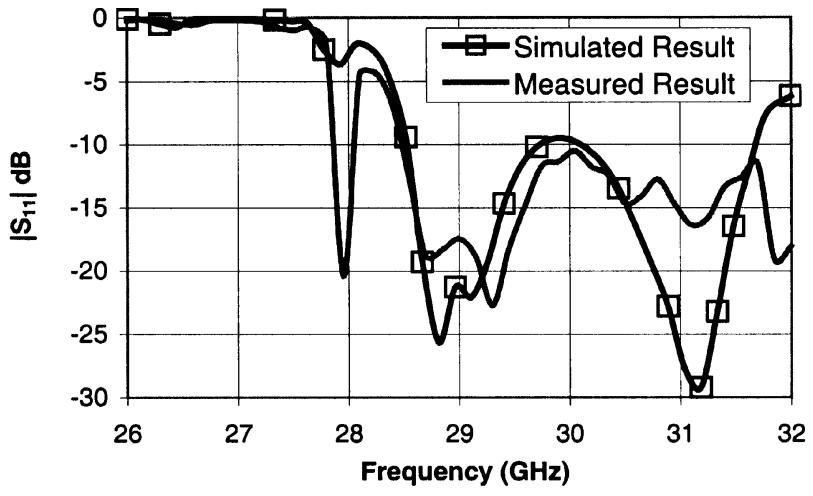

(a)

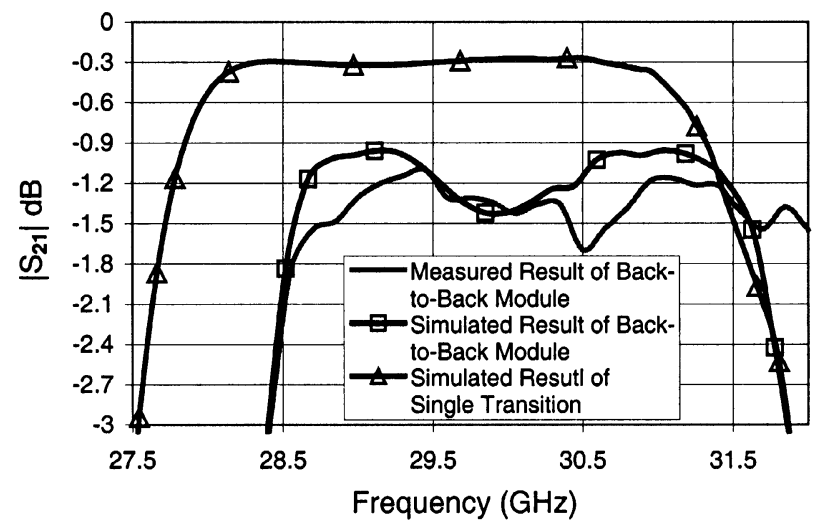

(b)

Fig. 11. Measured and simulated $S 21$ of the back-to-back module and EM simulated $S 21$ of the single transition module. (a) Magnitude of $S 11$ of the back-to-back configuration. (b) Magnitude of $S 21$ in the passband of the single transition and the back-to-back configuration.

that the insertion loss also includes insertion losses dissipated in a piece of 3-mm-long WR28 waveguide and a piece of 5-mm laminated waveguide.

\section{CONCLUSIONS}

A broad-band and compact laminated waveguide to air-filled waveguide transition has been proposed and fully investigated both theoretically and experimentally. The transition configuration is tailored to multilayer structures and can be easily integrated with any laminated waveguide integrated module for high-volume productions. In order to further interpret the transition configuration and provide a design guideline, an equivalent-circuit model is also proposed. The simulated results of the equivalent-circuit model and that of a full-wave EM model are very consistent with respect to the experimental results. The working principle of the transition interpreted by a pair of two-pole coupled resonators can also be used in designing other types of transition.

With its attractive features, the transition will be widely used in various wireless and radar systems where an integrated module with a laminated waveguide interface needs to be interconnected with an air-filled waveguide interfaced module.

\section{ACKNOWLEDGMENT}

The authors are very grateful to the LTCC Division, National Semiconductor Corporation, Irvine, CA, for providing LTCC prototyping support. The authors also extend many thanks Dr. M. Ehlert, National Semiconductor Corporation, for his great effort in building the LTCC parts.

\section{REFERENCES}

[1] F. Shigeki, "Waveguide line,” Japan Patent 06-053 711, Feb. 25, 1994.

[2] H. Uchimura, T. Takenoshita, and M. Fujii, "Development of a laminated waveguide," IEEE Trans. Microwave Theory Tech., vol. 46, pp. 2438-2443, Dec. 1998.

[3] H. Uchimura and T. Takenoshita, "Wiring board equipped with a line for transmitting a high frequency signal," U.S. Patent 5982 256, Nov. 9 , 1999.

[4] J. Hirokawa and M. Ando, "Efficiency of 76-GHz post-wall waveguide-fed parallel-plate slot arrays," IEEE Trans. Antennas Propagat., vol. 48, pp. 1742-1745, Nov. 2000.

[5] H. Uchimura and T. Takenoshita, "A ceramic planar $77 \mathrm{GHz}$ antenna array," in IEEE MTT-S Int. Microwave Symp. Dig., 1999, pp. 453-456.

[6] A. Piloto, K. Leahy, B. Flanick, and K. A. Zaki, "Waveguide filters having a layered dielectric structures," U.S. Patent 5382931 , Jan. 17, 1995.

[7] Y. Rong, K. A. Zaki, M. Hageman, D. Stevens, and J. Gipprich, "Low temperature cofired ceramic (LTCC) ridge waveguide bandpass chip filters," IEEE Trans. Microwave Theory Tech., vol. 47, pp. 2317-2324, Dec. 1999.

[8] D. Deslandes and K. Wu, "Integrated microstrip and rectangular waveguide in planar form," IEEE Microwave Wireless Comp. Lett., vol. 11, pp. 68-70, Feb. 2001.

[9] D. Deslandes and K. Wu, "Integrated transition of coplanar to rectangular waveguides," in IEEE MTT-S Int. Microwave Symp. Dig., 2001, pp. 619-622.

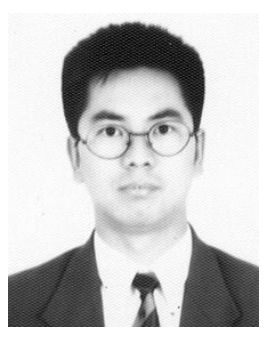

Yong Huang received the B.S. degree in electrical engineering from the East China Normal University, Shanghai, China, in 1992, the M.E. degree in electrical engineering from Xidian University, Xi'an, China, in 1999, and is currently working toward the $\mathrm{Ph} . \mathrm{D}$. degree in electronic engineering at the Chinese University of Hong Kong, Shatin, Hong Kong.

From 1992 to 2001, he was with the Antenna and Microwave Division, Xi' an Research Institute of Navigation Technology, Xi'an, China, where he was an Engineer. During that time, he conducted research in the areas of phased-array antenna design and frequency synthesizer design and was the Deputy Director of the division beginning in 1999. His current research interests include phased-array antenna design, novel integrated millimeter-wave system and components, and LTCC RF integrated-circuit design.

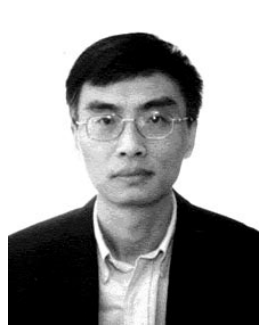

Ke-Li Wu (M'90-SM'96) received the B.S. and M.Eng. degrees from the Nanjing University of Science and Technology, Nanjing, China, in 1982 and 1985, respectively, and the Ph.D. degree from Laval University, Quebec, QC, Canada, in 1989.

From 1989 to 1993 , he was with the Communications Research Laboratory, McMaster University, Montreal, QC, Canada. In March 1993, he joined the Corporate Research and Development Division, Com Dev International, where he had been a Principle Member of Technical Staff in charge of developing advanced EM design software for various microwave subsystems. Since October 1999, he has been with the Department of Electronic Engineering, The Chinese University of Hong Kong, Shatin, Hong Kong, where he is a Professor. He has been published widely in the areas of EM modeling and microwave and antenna engineering. He holds one Canadian patent and one U.S. patent. His current research interests include all aspects related to LTCC multichip modules (MCMs) from EM modeling and design methodology to various applications.

Dr. Wu was a recipient of 1992 URSI Young Scientist Award. He was also the recipient of the 1993 Industry Feedback Award presented by the Telecommunication Research Institute of Ontario and the 1998 Com Dev Achievement Award. 